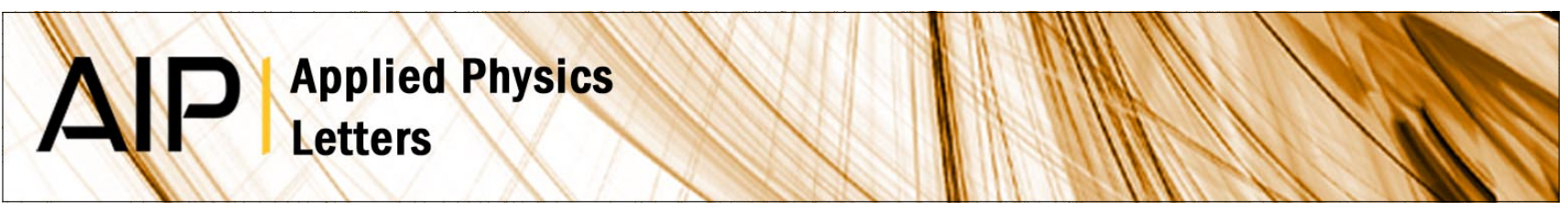

\title{
Epitaxial growth of europium monoxide on diamond
}

A. Melville, T. Mairoser, A. Schmehl, M. Fischer, S. Gsell et al.

Citation: Appl. Phys. Lett. 103, 222402 (2013); doi: 10.1063/1.4833550

View online: http://dx.doi.org/10.1063/1.4833550

View Table of Contents: http://apl.aip.org/resource/1/APPLAB/v103/i22

Published by the AIP Publishing LLC.

Additional information on Appl. Phys. Lett.

Journal Homepage: http://apl.aip.org/

Journal Information: http://apl.aip.org/about/about_the_journal

Top downloads: http://apl.aip.org/features/most_downloaded

Information for Authors: http://apl.aip.org/authors
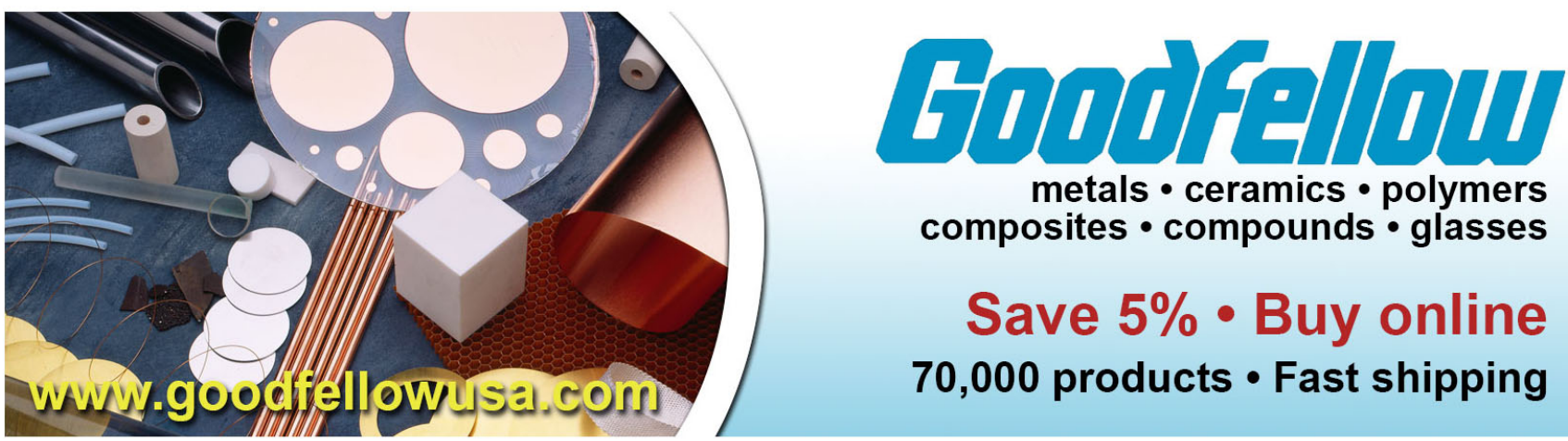


\title{
Epitaxial growth of europium monoxide on diamond
}

\author{
A. Melville, ${ }^{1}$ T. Mairoser, ${ }^{2}$ A. Schmehl, ${ }^{2}$ M. Fischer, ${ }^{3}$ S. Gsell, ${ }^{3}$ M. Schreck, ${ }^{3}$ \\ D. D. Awschalom, ${ }^{4}$ T. Heeg, ${ }^{1}$ B. Holländer, ${ }^{5}$ J. Schubert, ${ }^{5}$ and D. G. Schlom ${ }^{1,6, a)}$ \\ ${ }^{1}$ Department of Materials Science and Engineering, Cornell University, Ithaca, New York 14853, USA \\ ${ }^{2}$ Zentrum für elektronische Korrelationen und Magnetismus, Universität Augsburg, Universitätsstraße 1, \\ 86159 Augsburg, Germany \\ ${ }^{3}$ Institut für Physik, Universität Augsburg, D-86135 Augsburg, Germany \\ ${ }^{4}$ Institute for Molecular Engineering, University of Chicago, Chicago, Illinois 60637, USA \\ ${ }^{5}$ Peter Grünberg Institute, PGI9-IT, JARA-FIT, Research Centre Jülich, D-52425 Jülich, Germany \\ ${ }^{6}$ Kavli Institute at Cornell for Nanoscale Science, Ithaca, New York 14853, USA
}

(Received 3 September 2013; accepted 10 November 2013; published online 25 November 2013)

\begin{abstract}
We report the epitaxial integration of phase-pure $\mathrm{EuO}$ on both single-crystal diamond and on epitaxial diamond films grown on silicon utilizing reactive molecular-beam epitaxy. The epitaxial orientation relationship is (001) $\mathrm{EuO} \|(001)$ diamond and [110] $\mathrm{EuO} \|$ [100] diamond. The $\mathrm{EuO}$ layer is nominally unstrained and ferromagnetic with a transition temperature of $68 \pm 2 \mathrm{~K}$ and a saturation magnetization of $5.5 \pm 0.1$ Bohr magnetons per europium ion on the single-crystal diamond, and a transition temperature of $67 \pm 2 \mathrm{~K}$ and a saturation magnetization of $2.1 \pm 0.1 \mathrm{Bohr}$ magnetons per europium ion on the epitaxial diamond film. (C) 2013 AIP Publishing LLC. [http://dx.doi.org/10.1063/1.4833550]
\end{abstract}

Diamond has many desirable properties including high breakdown strength and unparalleled thermal conductivity. ${ }^{1}$ This makes it a promising substrate for high power and high frequency applications. Furthermore, diamond doped with nitrogen contains nitrogen-vacancy impurity centers capable of accommodating a long-lived electron spin state, which can be optically excited as well as optically read-out. ${ }^{2}$ As a result, diamond is capable of single-photon emissions from a specific spin state, making it a compelling platform for solidstate spin-based electronics at room temperature. ${ }^{2}$

Integrating this multipurpose substrate with functional oxide materials is a natural next step, as it can take advantage of the full range of properties found in oxide materials, e.g., high dielectric constant, ferroelectricity, ferromagnetism, and even oxides that are simultaneously ferroelectric and ferromagnetic. ${ }^{3}$ To date oxide materials have been minimally integrated with diamond for use in surface acoustic wave devices, ${ }^{4-6}$ or as a buffer layer for other oxide materials. $^{7}$ There has only been one report of the epitaxial growth of an oxide on diamond and that is (0001) $\mathrm{ZnO}$ on (111) diamond. ${ }^{4}$ In this Letter, we demonstrate the epitaxial integration of the ferromagnet $\mathrm{EuO}$ with (001) diamond single crystals and with epitaxial (001) diamond films on silicon.

Europium oxide $(\mathrm{EuO})$ is a ferromagnetic semiconductor with a spin-polarization of at least $96 \%, 8,9$ giving it the second highest spin-polarization of all known materials after $\mathrm{CrO}_{2}{ }^{10}$ Furthermore, doped $\mathrm{EuO}$ has a metal-to-insulator transition (MIT) involving a change in resistivity of up to 13 orders of magnitude ${ }^{11}$ and exhibits colossal magneto-resistance (CMR) up to 6 orders of magnitude. ${ }^{12}$ The MIT and CMR occur around the Curie temperature $\left(T_{\mathrm{C}}\right)$, which is $69 \mathrm{~K}$ in bulk EuO. ${ }^{13} T_{\mathrm{C}}$ can be enhanced dramatically by doping with trivalent cations $\mathrm{s}^{8,9,14-21}$ or by introducing oxygen vacancies

\footnotetext{
${ }^{\text {a) }}$ Author to whom correspondence should be addressed. Electronic mail schlom@cornell.edu
}

$\left(\mathrm{EuO}_{1-x}\right){ }^{22-25}$ Theorists predict that a $T_{\mathrm{C}}$ of $\sim 200 \mathrm{~K}$ is possible by combining doping and compressive strain. ${ }^{26}$

The epitaxial integration of $\mathrm{EuO}$ with modern semiconductor materials, i.e., $\mathrm{Si}^{8,27} \mathrm{GaN}^{8}$ and $\mathrm{GaAs}^{28}$ has already been demonstrated. The lattice mismatch between (001) diamond and (001) $\mathrm{EuO}(\sim 2 \%)$ corresponds to cube-on-cube growth where the $\mathrm{EuO}$ grows $45^{\circ}$ rotated in-plane compared to the underlying diamond substrate. The diamond surface mesh is illustrated in the top left of Fig. 1, and the arrangement of $\mathrm{EuO}$ on diamond resulting in the $\sim 2 \%$ lattice mismatch is illustrated in the top right of Fig. 1. This relatively small lattice mismatch compares favorably to the lattice mismatch between $\mathrm{EuO}$ and $\mathrm{Si}, \mathrm{GaN}$, or GaAs. As the lattice constant of diamond is smaller than that of $\mathrm{EuO}$, a commensurate EuO film on diamond (if it could be achieved) would be in biaxial compression and an enhancement of $T_{\mathrm{C}}$ from $69 \mathrm{~K}$ to $\sim 80 \mathrm{~K}$ would be expected in undoped $\mathrm{EuO}$ from first-principles calculations. ${ }^{29}$ Thus, the epitaxial integration of $\mathrm{EuO}$ with diamond could potentially result in coherently strained EuO films exhibiting a higher $T_{\mathrm{C}}$ than epitaxial EuO films grown on $\mathrm{Si}^{8,27} \mathrm{GaN},{ }^{8}$ and $\mathrm{GaAs}^{28}{ }^{28}$ while still allowing favorable substrate qualities such as long spin lifetimes for injected electrons ${ }^{30}$ or a long-lived electron spin state. ${ }^{2}$

$10 \mathrm{~mm} \times 10 \mathrm{~mm}$ epitaxial (001)-oriented diamond films were grown by CVD on (001) Si utilizing iridium metal and yttria-stabilized zirconia (YSZ) buffer layers, ${ }^{31} 5 \mathrm{~mm} \times 5 \mathrm{~mm}$ $\times 1 \mathrm{~mm}$ diamond single crystals grown by CVD with (001) surfaces were obtained commercially. ${ }^{32}$ The EuO thin films on the epitaxial diamond films were grown in a Veeco 930 molecular-beam epitaxy (MBE) system, and the EuO thin films on single-crystal diamond were grown in a Veeco Gen10 MBE system. A cryoshroud cooled with liquid nitrogen was employed in both MBE chambers to reduce the chamber background pressure to less than $1 \times 10^{-8}$ Torr. EuO films on both types of (001) diamond substrates were grown via reactive oxide $\mathrm{MBE}$ in an adsorption-controlled growth regime ${ }^{33}$ to ensure high crystalline quality and stoichiometric films. The 


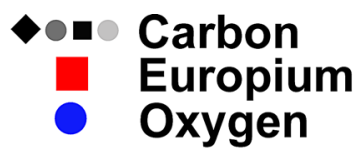

(001) Diamond (001) EuO
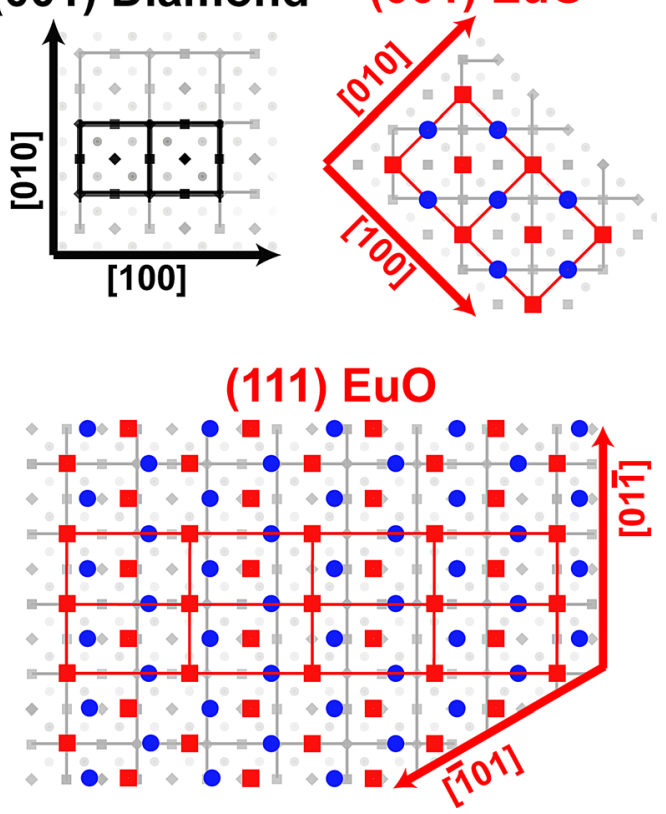

FIG. 1. Diagram showing the epitaxial orientation relationship between the surface mesh of the (001) EuO film and underlying (001) diamond substrate. The top left image is the diamond surface mesh with two unit cells highlighted. The top right image corresponds to the epitaxial alignment of EuO on diamond corresponding to $\sim 2 \%$ lattice mismatch, with two EuO unit cells highlighted. This orientation relationship is (001)[110] EuO \| (001)[100] diamond and corresponds to a $\sigma_{\text {EuO }} 1: \sigma_{\text {diamond }} 2$ interface. The bottom image corresponds to the epitaxial alignment of $\mathrm{EuO}$ on diamond with less strain, but with a near-coincident site lattice, with four $\mathrm{EuO}$ unit cells highlighted. Here, the epitaxial alignment is (111)[011] EuO $\|(001)[010]$ diamond. This corresponds to a $\sigma_{\mathrm{EuO}} 4: \sigma_{\text {diamond }} 7$ interface.

europium flux, measured by a quartz crystal microbalance, was set to $1.1 \times 10^{14}$ atoms $/\left(\mathrm{cm}^{2} \mathrm{~s}\right)$. This flux was roughly double that of the EuO film growth rate, as determined by measuring the areal density of europium atoms in calibration EuO samples using Rutherford backscattering spectrometry (RBS), ensuring an overabundance of europium during growth. Under such conditions, the EuO growth rate is limited by the oxygen flux. ${ }^{33}$ The oxygen flux was established by flowing oxygen through a piezoelectric leak valve set at a constant voltage. This resulted in a slight increase in the background pressure during growth that increased gradually over time, but was never more than $1 \times 10^{-8}$ Torr. After growth, the films were capped with $\sim 100 \mathrm{~nm}$ of aluminum or $\sim 20 \mathrm{~nm}$ of amorphous silicon to prevent further oxidation of the EuO films and enable their ex situ characterization. Structural characterization was performed after growth using four-circle X-ray diffraction (XRD) and X-ray reflectivity utilizing $\mathrm{Cu} K_{\alpha}$ radiation. Magnetic properties and the Curie temperature were determined using superconducting quantum interference device (SQUID) magnetometry.

For the growth of EuO on epitaxial diamond films with a diamond surface mesh as shown in the upper left of Fig. 1, the EuO grew with two epitaxial orientations under growth conditions similar to those used to grow highquality $\mathrm{EuO}$ on $\mathrm{YAlO}_{3}\left(T=590{ }^{\circ} \mathrm{C}\right) .^{33}$ The dominant orientation as determined by XRD measurements was (111) EuO $\|$ (001) diamond with [011] EuO || [010] diamond as illustrated in the bottom image of Fig. 1, and the secondary orientation was (001) EuO || (001) diamond also with [110] $\mathrm{EuO} \|$ [100] diamond as illustrated in the top right image of Fig. 1.

The optimal growth conditions for growing (001)-oriented $\mathrm{EuO}$ on epitaxial diamond films was achieved by first growing two monolayers to establish the orientation at lower growth temperatures $\left(350^{\circ} \mathrm{C}<T<400^{\circ} \mathrm{C}\right)$. Once the orientation was seeded, the growth temperature was ramped up to $650^{\circ} \mathrm{C}$ (while continuing growth) for better crystallinity. In this case, only the orientation relationship (001)[110] EuO || (001)[100] diamond was found as illustrated in the top right of Fig. 1. This relatively low starting temperature was established as the lower limit of the adsorption-controlled growth regime by depositing europium in the absence of oxygen on a hot substrate for an hour and confirming absence of an accumulation layer of europium by RBS.

For the growth of EuO on the single-crystal diamond, the EuO also grew with two epitaxial orientations with a dominant orientation as determined by XRD measurements of (111) EuO || (001) diamond with [011] EuO || [010] diamond under the adsorption-controlled growth conditions similar to those used to grow high-quality (001) EuO on (110) $\mathrm{YAlO}_{3}$ $\left(T=590{ }^{\circ} \mathrm{C}\right){ }^{33}$ In contrast to growths on the epitaxial diamond films, however, films grown at lower growth temperatures $\left(T<590^{\circ} \mathrm{C}\right)$ on single-crystal diamonds were almost exclusively composed of the epitaxial orientation of (111)

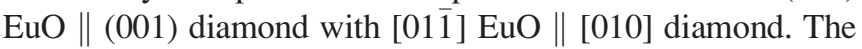
optimal growth conditions for growing (001) EuO on single-crystal diamond was to grow at higher growth temperatures $\left(T=760^{\circ} \mathrm{C}\right)$, where only the orientation relationship (001)[110] $\mathrm{EuO} \|$ (001)[100] diamond was found.

The difference in EuO growth conditions could be related to differences in structural perfection of the epitaxial diamond film vs. single-crystal diamond (diamond film 004 peak full width at half-maximum (FWHM) of $0.2^{\circ}$ as compared to the single-crystal diamond 004 peak FWHM of $\left.0.005^{\circ}\right)$ or the difference in surface roughness $\left(R_{\mathrm{RMS}}(\right.$ diamond film $)=13.3 \mathrm{~nm}$ as compared to $\mathrm{R}_{\mathrm{RMS}}$ ( ingle-crystal diamond $)=1 \mathrm{~nm}$ ).

Epitaxial orientation relationships that change with growth temperature have been seen in other oxide systems, e.g., in the epitaxial growth of $\mathrm{YBa}_{2} \mathrm{Ca}_{3} \mathrm{O}_{7-\delta}$ on YSZ. ${ }^{34-36} \mathrm{In}$ that system the out-of-plane orientation relationship, (001) $\mathrm{YBa}_{2} \mathrm{Cu}_{3} \mathrm{O}_{7} \|$ (001) YSZ, was found to remain constant, while the in-plane orientation relationship was observed to change with growth temperature. ${ }^{36}$ At low substrate temperature, the dominant in-plane epitaxial orientation relationship observed was [100] $\mathrm{YBa}_{2} \mathrm{Ca}_{3} \mathrm{O}_{7-\delta} \|$ [100] $\mathrm{YSZ}$ while films deposited at high temperature had [110] $\mathrm{YBa}_{2} \mathrm{Ca}_{3} \mathrm{O}_{7-\delta} \|$ [100] YSZ. ${ }^{36}$

The (111) EuO || (001) diamond orientation has a slightly lower mismatch, $+1.8 \%$ for [2 $\overline{1} \overline{1}]$ EuO $\|$ [100] diamond and $-2.1 \%$ for $[01 \overline{1}] \mathrm{EuO} \|$ [010] diamond, than the expected (001) $\mathrm{EuO} \|$ (001) diamond orientation, which has a lattice mismatch of $-2.1 \%$ for both [110] EuO $\|$ [100] diamond and [110] EuO || [010] diamond. Although better lattice matched, the (111) EuO || (001) diamond orientation has a larger nearcoincident site surface mesh cell corresponding to a 
Coincident Site Lattice/Displacement Shift Complete (CSL/DSC) model $^{37}$ interface of $\sigma_{\mathrm{EuO}} 4: \sigma_{\text {diamond }} 7$ as shown at the bottom of Fig. 1. And though films with the better lattice-matched orientation are epitaxial, the (111) EuO is undesirable, as it contains four distinct in-plane twin variants, resulting in a multitude of twin boundaries. The (001) EuO $\|$ (001) diamond orientation is preferred as there is only one in-plane orientation and the films are free of twin boundaries. Furthermore, this latter orientation relationship has a coincident site lattice, corresponding to a CSL/DSC model interface of $\sigma_{\mathrm{EuO}} 1: \sigma_{\text {diamond }} 2$ as shown at the top right of Fig. 1.

Having established the optimal conditions for the growth of (001) $\mathrm{EuO}$ on both single-crystal diamond and on epitaxial diamond films, the remainder of the paper will focus on these (001) EuO films. Reflection high-energy electron diffraction (RHEED) images were taken of both types of EuO films. Figures 2(a) and 2(b) show the RHEED images with the incident electron beam along the [100] and [110] azimuths, respectively, of (001) EuO on the epitaxial diamond films, and Figs. 2(c) and 2(d) show the RHEED images along the [100] and [110] azimuths, respectively, of (001) $\mathrm{EuO}$ on the single-crystal diamond. In all four cases, there is no evidence of other phases or orientations. The transmission diffraction pattern evident in the (001) EuO on single-crystal diamond (Figs. 2(c) and 2(d)) suggest that the film is atomically rough.

The $\theta-2 \theta$ scans in Figs. 3(a) and 3(b) reveal only $00 \ell$ $\mathrm{EuO}$ peaks and capping layer peaks, indicating that the films are single phase with the desired (001) EuO out-of-plane orientation. From calculations of the out-of-plane lattice constant expected for a commensurate ( $2.1 \%$ biaxial compression) $\mathrm{EuO}$ film on diamond utilizing the elastic coefficients of $\mathrm{EuO}^{38}$ we expected an out-of-plane lattice constant of $c=5.188 \AA$ as compared to the bulk-value of $c=5.141 \AA .{ }^{39}$

Nelson-Riley analysis ${ }^{40}$ on the 002, 004, and $006 \mathrm{EuO}$ peaks on the epitaxial diamond film, however, yields $c=5.137 \pm 0.001 \AA$, and Nelson-Riley analysis on the 002 ,
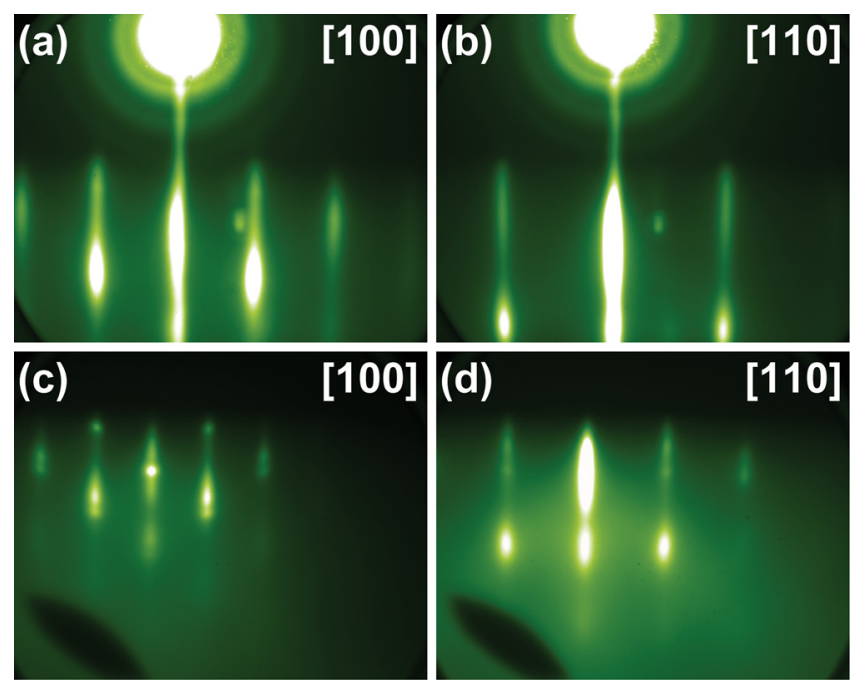

FIG. 2. RHEED images of a $25 \mathrm{~nm}$ thick EuO film grown at $350^{\circ} \mathrm{C}<T_{\text {sub }}$ $<400^{\circ} \mathrm{C}$ for two monolayers then ramped to $T_{\text {sub }}=650^{\circ} \mathrm{C}$ on an epitaxial diamond film along the (a) [100] azimuth and the (b) [110] azimuth of the EuO thin film. RHEED images of a $37 \mathrm{~nm}$ thick EuO film grown at $T_{\text {sub }}=760^{\circ} \mathrm{C}$ on a single-crystal diamond along the (c) [100] azimuth and the (d) [110] azimuth.
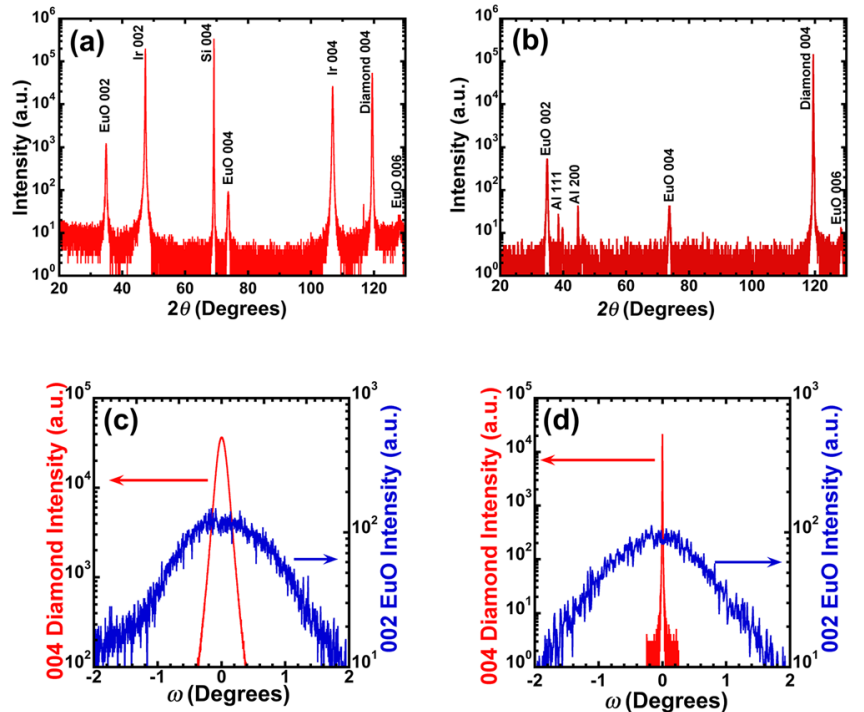

FIG. 3. (a) A $\theta-2 \theta$ scan of the same $25 \mathrm{~nm}$ thick EuO film grown on an epitaxial diamond film as shown in Figs. 2(a) and 2(b) reveals phase-pure EuO with only an (001) out-of-plane orientation. (b) A $\theta-2 \theta$ scan of the same $37 \mathrm{~nm}$ thick EuO film grown on a single-crystal diamond as shown in Figs. 2(c) and 2(d) reveals phase-pure EuO with only an (001) out-of-plane orientation. There is no indication of europium metal or europium oxides with higher oxygen content in either film. (c) Rocking curve comparison between $002 \mathrm{EuO}$ and 004 diamond of the same EuO film grown on an epitaxial diamond film as shown in Fig. 3(a). (d) Rocking curve comparison between $002 \mathrm{EuO}$ and 004 diamond of the same EuO film grown on a single-crystal diamond as shown in Fig. 3(b).

004, and $006 \mathrm{EuO}$ peaks on single-crystal diamond yields $c=5.135 \pm 0.001 \AA$. Our epitaxial $(001) \mathrm{EuO}$ films are clearly not commensurate. The reduced out-of-plane spacing from that of bulk $\mathrm{EuO}$ indicates that the films are under slight biaxial tensile strain, likely due to thermal strain imparted during cooling after growth due to the much larger linear thermal expansion coefficient of EuO compared to that of the single-crystal diamond and silicon substrates over relevant temperatures. ${ }^{41-43}$

Rocking curve measurements of the film and underlying substrate were compared using the $\mathrm{EuO} 002$ peak and diamond 004 peak. For the $\mathrm{EuO}$ on the epitaxial diamond film, Fig. 3(c), the EuO 002 peak has a FWHM of $1.2^{\circ}$ in omega compared to the diamond 004 peak FWHM of $0.17^{\circ}$. For the $\mathrm{EuO}$ on single-crystal diamond, Fig. 3(d), the EuO 002 peak has a FWHM of $1.8^{\circ}$ in omega compared to the diamond 004 peak FWHM of $0.005^{\circ}$. The large EuO peak FWHM further suggests that the films contain high densities of dislocations and are therefore not commensurate with the underlying substrates. Since the films were not commensurately strained, we could not test the prediction of an enhanced $T_{\mathrm{C}}$ in compressively strained $\mathrm{EuO}^{26}$

Figure 4(a) shows $\phi$-scans of the $111 \mathrm{Si}, 111 \mathrm{Ir}, 111$ diamond, and $222 \mathrm{EuO}$ peaks of the $\mathrm{EuO}$ film on the epitaxial diamond film. As expected the diamond, iridium, YSZ, and silicon layers are arranged cube-on-cube with (001)[100] diamond $\|(001)[100]$ Ir $\|(001)[100]$ YSZ $\|(001)[100] \mathrm{Si}^{31}$ $\mathrm{EuO}$ is also arranged cube-on-cube, but rotated $45^{\circ}$ with respect to the underlying diamond film as discussed earlier. The $45^{\circ}$ rotation is denoted by the displacement of the four 222 peaks of EuO relative to the four 111 diamond peaks. The YSZ layer could not be resolved by XRD due to its 
small thickness. Together the $\theta-2 \theta$ scan and $\phi$-scans indicate an epitaxial film of $\mathrm{EuO}$ on diamond with an orientation relationship of (001)[110] EuO || (001)[100] diamond. A schematic depicting the orientation relationship between the layers in this epitaxial heterostructure is shown in Fig. 4(b).

Figure 4(c) shows $\phi$-scans of the $111 \mathrm{EuO}$ and 111 diamond reflections of the $\mathrm{EuO}$ on single-crystal diamond. $\mathrm{EuO}$ is arranged cube-on-cube, again rotated $45^{\circ}$ with respect to the underlying diamond. The $45^{\circ}$ rotation is denoted by the displacement of the four 111 peaks of EuO relative to the four 111 diamond peaks. Together the $\theta-2 \theta$ scan and $\phi$-scans indicate an epitaxial film of EuO on diamond with an orientation relationship of (001)[110] EuO $\|$ (001)[100] diamond. The $d$ spacing of the (110) EuO plane was $d_{110}=3.647 \pm 0.003 \AA$, as calculated from the measured $\theta-2 \theta$ positions of multiple reflections from the (111) EuO planes. This value is larger than the bulk value $\left(d_{110}=3.635 \AA\right),{ }^{39}$ providing further

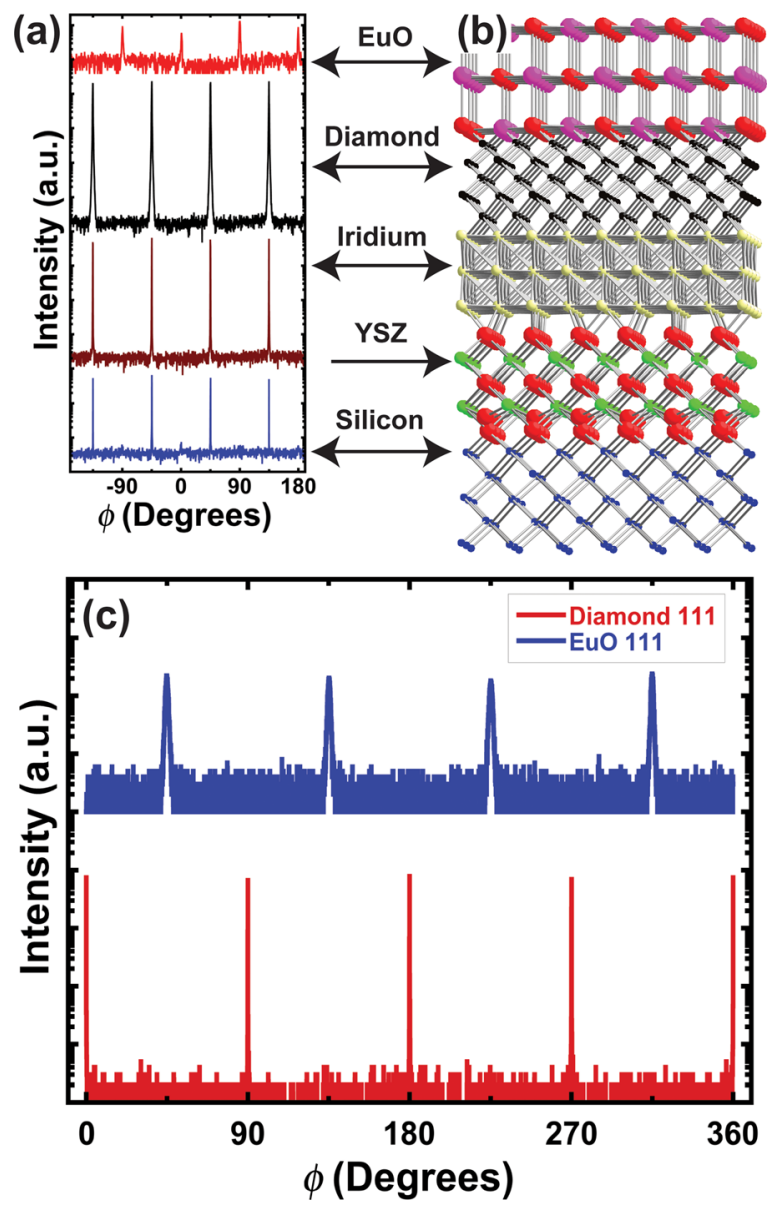

FIG. 4. (a) Azimuthal $\phi$-scans of the same $25 \mathrm{~nm}$ thick EuO film grown on an epitaxial diamond film as shown in Fig. 3(a) for the $111 \mathrm{Si}, 111 \mathrm{Ir}, 111 \mathrm{di}$ amond, and $222 \mathrm{EuO}$ diffraction peaks at $\chi=54.7^{\circ}$, where $\chi=0^{\circ}$ aligns the diffraction vector perpendicular to the plane of the substrate. $\phi=0^{\circ}$ corresponds to the in-plane component of the diffraction vector aligned parallel to the [100] direction of the (001) EuO film. The scans are offset for clarity. The positions of the phi peaks indicate that the orientation relationship between the layers is (001)[110] EuO || (001)[100] diamond || (001)[100] Ir \| (001)[100] Si. (b) An atomic model illustrates the orientation relationship between all components in the heterostructure. (c) Azimuthal $\phi$-scans of the same $37 \mathrm{~nm}$ thick EuO film grown on a single-crystal diamond as shown in Fig. 3(b) for the 111 diamond and $111 \mathrm{EuO}$ diffraction peaks at $\chi=54.7^{\circ}$. The scans are offset for clarity. The positions of the phi peaks indicate that the orientation relationship between the layers is (001)[110] EuO \| (001)[100] diamond. evidence that the film is under tensile strain as a result of the lattice being relaxed at the high growth temperature and clamped to the substrate during cooling to room temperature. $^{43}$ The observed tensile strain, $0.3 \pm 0.08 \%$, is expected to reduce the $T_{\mathrm{C}}$ of $\mathrm{EuO}$ by about $1.5 \mathrm{~K}$ according to first-principles calculations. ${ }^{29}$

Magnetic measurements were performed in zero applied field and the Curie temperature was determined from the derivative of the magnetization with respect to temperature. ${ }^{44}$ The $T_{\mathrm{C}}$ was found to be $67 \pm 2 \mathrm{~K}$ for $\mathrm{EuO}$ grown on the epitaxial diamond film (Fig. 5(a)) and $68 \pm 2 \mathrm{~K}$ for EuO grown on the single-crystal diamond (Fig. 5(b)), which is within experimental error of the expected Curie temperature of EuO given the slight $T_{\mathrm{C}}$ shift from the observed tensile strain. ${ }^{26,29}$ Magnetic hysteresis of the films reveals a saturation magnetization of $2.1 \pm 0.1 \mu_{\mathrm{B}}$ per europium ion and a coercive field of $50 \pm 50 \mathrm{G}$ at $40 \mathrm{~K}$ for the EuO grown on the epitaxial diamond film (Fig. 5(c)) and a saturation magnetization of $5.5 \pm 0.1 \mu_{\mathrm{B}}$ per europium ion and a coercive field of $105 \pm 10 \mathrm{G}$ at $5 \mathrm{~K}$ for the EuO grown on single-crystal diamond (Fig. 5(d)). At $5 \mathrm{~K}$, the EuO grown on the epitaxial diamond film has a maximum magnetization at $30000 \mathrm{G}$ of $3.7 \pm 0.1 \mu_{\mathrm{B}}$ per europium ion. These values are considerably lower than the $6.9 \mu_{\mathrm{B}}$ per europium ion found in bulk $\mathrm{EuO}$ and in high-quality $\mathrm{EuO}$ epitaxial films, ${ }^{8,9,17,28,33,39}$ and is indicative of our films on diamond not yet being the quality of epitaxial $\mathrm{EuO}$ films grown on $\mathrm{YAlO}_{3},{ }^{8,33} \mathrm{LuAlO}_{3},{ }^{29} \mathrm{Si}^{8}$, and $\mathrm{GaAs}^{28}$

In summary, the epitaxial integration of ferromagnetic $\mathrm{EuO}$ on epitaxial diamond films and single-crystal diamond
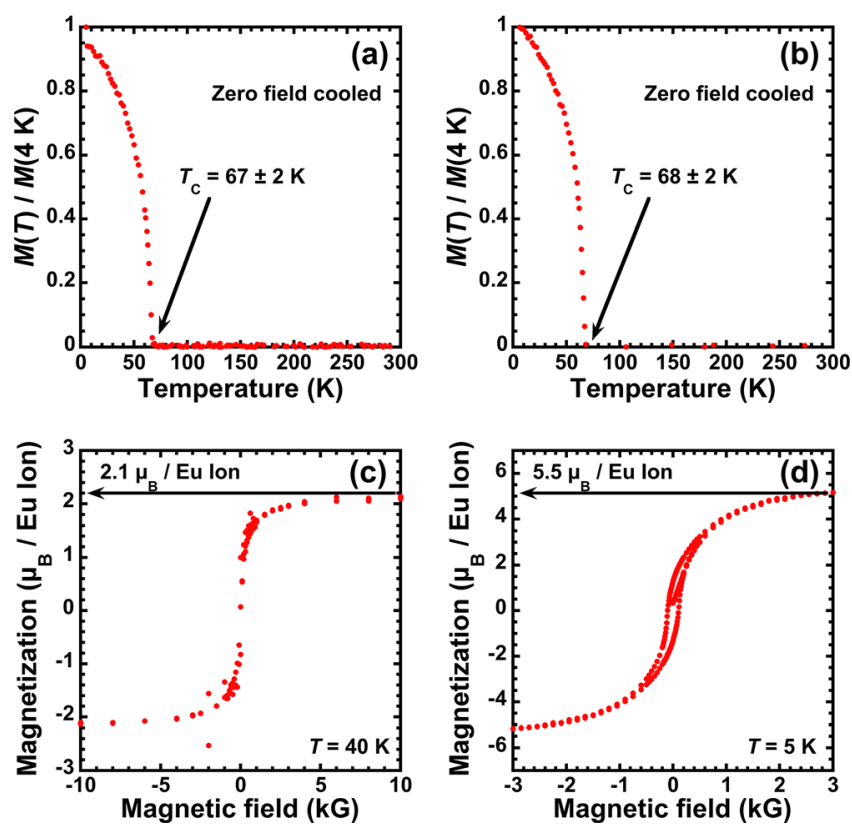

FIG. 5. Magnetization as a function of temperature of (a) the same $25 \mathrm{~nm}$ thick EuO film grown on an epitaxial diamond film as shown in Fig. 3(a) revealing a $T_{\mathrm{C}}$ of $67 \pm 2 \mathrm{~K}$ and (b) the same $37 \mathrm{~nm}$ thick EuO film grown on a single-crystal diamond as shown in Fig. 3(b) revealing a $T_{\mathrm{C}}$ of $68 \pm 2 \mathrm{~K}$. (c) Magnetic hysteresis measurements show that the same $25 \mathrm{~nm}$ thick EuO film grown on epitaxial diamond film as shown in Fig. 3(a) is ferromagnetic with a coercive field of $50 \pm 50 \mathrm{G}$ and a saturation magnetization of $2.1 \pm 0.1$ Bohr magnetons per europium ion at $40 \mathrm{~K}$. (d) Magnetic hysteresis measurements show that the same $37 \mathrm{~nm}$ thick $\mathrm{EuO}$ film grown on single-crystal diamond as shown in Fig. 3(b) is ferromagnetic with a coercive field of $105 \pm 10 \mathrm{G}$ and a saturation magnetization of $5.5 \pm 0.1 \mathrm{Bohr}$ magnetons per europium ion at $5 \mathrm{~K}$. 
was achieved. The epitaxy of the EuO film on (001) diamond exhibited a temperature-dependent orientation as seen in other heteroepitaxy oxide systems. The magnetic hysteresis and Curie temperature of epitaxial EuO films on diamond are comparable to those of bulk EuO. ${ }^{13}$ Although the growth conditions used yielded relaxed $\mathrm{EuO}$ films, the predicted enhancement of $T_{\mathrm{C}}$ by compressive strain $^{26}$ motivates the achievement of commensurate EuO films on diamond-a challenge for future studies.

The work at Cornell and at the Institute for Molecular Engineering at the University of Chicago was supported by the AFOSR (Grant No. FA9550-10-1-0123). The work in Augsburg was supported by the DFG (Grant No. TRR 80) and the EC (oxIDes). AJM gratefully acknowledges support from the NSF IGERT program (NSF Award DGE-0654193). This work was performed in part at the Cornell NanoScale Facility, a member of the National Nanotechnology Infrastructure Network, which is supported by the National Science Foundation (Grant ECCS-0335765).

${ }^{1}$ Y. Gurbuz, O. Esame, I. Tekin, W. Kang, and J. Davidson, Solid-State Electron. 49, 1055-1070 (2005).

${ }^{2}$ D. D. Awschalom, R. J. Epstein, and R. Hanson, Sci. Am. 297, 84-91 (2007).

${ }^{3}$ J. H. Lee, L. Fang, E. Vlahos, X. Ke, Y. W. Jung, L. F. Kourkoutis, J.-W. Kim, P. J. Ryan, T. Heeg, M. Roeckerath, V. Goian, M. Bernhagen, R. Uecker, P. C. Hammel, K. M. Rabe, S. Kamba, J. Schubert, J. W. Freeland, D. A. Muller, C. J. Fennie, P. Schiffer, V. Gopalan, E. JohnstonHalperin, and D. G. Schlom, Nature 466, 954-958 (2010).

${ }^{4}$ A. Hachigo, H. Nakahata, K. Higaki, S. Fujii, and S.-I. Shikata, Appl. Phys. Lett. 65, 2556-2558 (1994).

${ }^{5}$ H. Lam, J. Cryst. Growth 268, 144-148 (2004).

${ }^{6}$ J. J. Chen, F. Zeng, D. M. Li, J. B. Niu, and F. Pan, Thin Solid Films 485, 257-261 (2005).

${ }^{7}$ S. M. Lee, H. Murakami, and T. Ito, Appl. Surf. Sci. 175-176, 517-524 (2001).

${ }^{8}$ A. Schmehl, V. Vaithyanathan, A. Herrnberger, S. Thiel, C. Richter, M. Liberati, T. Heeg, M. Röckerath, L. F. Kourkoutis, S. Mühlbauer, P. Böni, D. A. Muller, Y. Barash, J. Schubert, Y. Idzerda, J. Mannhart, and D. G. Schlom, Nature Mater. 6, 882-887 (2007).

${ }^{9}$ A. Melville, T. Mairoser, A. Schmehl, D. E. Shai, E. J. Monkman, J. W. Harter, T. Heeg, B. Holländer, J. Schubert, K. M. Shen, J. Mannhart, and D. G. Schlom, Appl. Phys. Lett. 100, 222101 (2012).

${ }^{10}$ A. Anguelouch, A. Gupta, G. Xiao, D. Abraham, Y. Ji, S. Ingvarsson, and C. Chien, Phys. Rev. B 64, 180408 (2001).

${ }^{11}$ G. Petrich, S. von Molnár, and T. Penney, Phys. Rev. Lett. 26, 885-888 (1971).

${ }^{12}$ Y. Shapira, S. Foner, and T. B. Reed, Phys. Rev. B 8, 2299 (1973).

${ }^{13}$ T. R. McGuire and M. W. Shafer, J. Appl. Phys. 35, 984-988 (1964).

${ }^{14}$ K. Y. Ahn, Appl. Phys. Lett. 17, 347-349 (1970).

${ }^{15}$ K. Lee and J. C. Suits, Phys. Lett. A 34, 141-142 (1971).

${ }^{16}$ K. Lee and J. C. Suits, IEEE Trans. Magn. 7, 391 (1971).

${ }^{17}$ R. Sutarto, S. Altendorf, B. Coloru, M. Moretti Sala, T. Haupricht, C, Chang, Z. Hu, C. Schüßler-Langeheine, N. Hollmann, H. Kierspel, J. Mydosh, H. Hsieh, H.-J. Lin, C. Chen, and L. Tjeng, Phys. Rev. B 80, 085308 (2009).

${ }^{18}$ H. Miyazaki, H. J. Im, K. Terashima, S. Yagi, M. Kato, K. Soda, T. Ito, and S. Kimura, Appl. Phys. Lett. 96, 232503 (2010).
${ }^{19}$ T. Mairoser, A. Schmehl, A. Melville, T. Heeg, L. Canella, P. Böni, W. Zander, J. Schubert, D. Shai, E. Monkman, K. Shen, D. G. Schlom, and J. Mannhart, Phys. Rev. Lett. 105, 257206 (2010).

${ }^{20}$ T. Mairoser, A. Schmehl, A. Melville, T. Heeg, W. Zander, J. Schubert, D. E. Shai, E. J. Monkman, K. M. Shen, T. Z. Regier, D. G. Schlom, and J. Mannhart, Appl. Phys. Lett. 98, 102110 (2011).

${ }^{21}$ P. Liu, J. Tang, J. A. Colón Santana, K. D. Belashchenko, and P. A. Dowben, J. Appl. Phys. 109, 07C311 (2011).

${ }^{22}$ K. Y. Ahn and M. W. Shafer, J. Appl. Phys. 41, 1260-1262 (1970).

${ }^{23}$ M. W. Shafer, J. B. Torrance, and T. Penney, J. Phys. Chem. Solids 33, 2251-2266 (1972).

${ }^{24}$ C. Llinares, J. P. Desfours, J. P. Nadai, C. Godart, A. Percheron, and J. C. Achard, Phys. Status Solidi A 25, 185-192 (1974).

${ }^{25}$ M. Barbagallo, N. Hine, J. Cooper, N.-J. Steinke, A. Ionescu, C. Barnes, C. Kinane, R. Dalgliesh, T. Charlton, and S. Langridge, Phys. Rev. B 81, 235216 (2010).

${ }^{26}$ N. Ingle and I. Elfimov, Phys. Rev. B 77, 121202 (2008).

${ }^{27}$ J. Lettieri, V. Vaithyanathan, S. K. Eah, J. Stephens, V. Sih, D. D. Awschalom, J. Levy, and D. G. Schlom, Appl. Phys. Lett. 83, 975-977 (2003).

${ }^{28}$ A. G. Swartz, J. Ciraldo, J. J. I. Wong, Y. Li, W. Han, T. Lin, S. Mack, J. Shi, D. D. Awschalom, and R. K. Kawakami, Appl. Phys. Lett. 97, 112509 (2010).

${ }^{29}$ A. Melville, T. Mairoser, A. Schmehl, T. Birol, T. Heeg, B. Holländer, J. Schubert, C. J. Fennie, and D. G. Schlom, Appl. Phys. Lett. 102, 062404 (2013).

${ }^{30}$ G. Balasubramanian, P. Neumann, D. Twitchen, M. Markham, R. Kolesov, N. Mizuochi, J. Isoya, J. Achard, J. Beck, J. Tissler, V. Jacques, P. R. Hemmer, F. Jelezko, and J. Wrachtrup, Nature Mater. 8, 383-387 (2009).

${ }^{31}$ S. Gsell, T. Bauer, J. Goldfuß, M. Schreck, and B. Stritzker, Appl. Phys. Lett. 84, 4541-4543 (2004).

${ }^{32}$ Element Six, N. V., Cuijk, The Netherlands.

${ }^{33}$ R. W. Ulbricht, A. Schmehl, T. Heeg, J. Schubert, and D. G. Schlom, Appl. Phys. Lett. 93, 102105 (2008).

${ }^{34}$ D. K. Fork, S. M. Garrison, M. Hawley, and T. H. Geballe, J. Mater. Res. 7, 1641-1651 (1992).

${ }^{35}$ J. A. Alarco, G. Brorsson, G. Ivanov, P. Nilsson, E. Olsson, and M. Löfgren, Appl. Phys. Lett. 61, 723-725 (1992).

${ }^{36}$ S. M. Garrison, N. Newman, F. Cole, K. Char, and R. W. Barton, Appl. Phys. Lett. 58, 2168-2170 (1991).

${ }^{37}$ R. W. Balluffi, A. Brokman, and A. H. King, Acta Metall. 30, 1453-1470 (1982).

${ }^{38}$ Y. Shapira and T. B. Reed, AIP Conf. Proc. 5, 837-839 (1972).

${ }^{39}$ B. T. Matthias, R. M. Bozorth, and J. H. Van Vleck, Phys. Rev. Lett. 7, 160-161 (1961).

${ }^{40}$ J. B. Nelson and D. P. Riley, Proc. Phys. Soc. 57, 160-178 (1945).

${ }^{41}$ G. A. Slack and S. F. Bartram, J. Appl. Phys. 46, 89-98 (1975).

${ }^{42}$ D. Taylor, Trans. J. Br. Ceram. Soc. 83, 5-9 (1984).

${ }^{43}$ For the growth of EuO on epitaxial diamond film substrates, the thermal expansion mismatch between EuO and silicon would lead to an in-plane tensile strain of $0.6 \%$ and an out-of-plane lattice spacing of $5.127 \AA$ for the $25^{\circ} \mathrm{C}$ XRD measurement, assuming that the EuO film is fully relaxed at growth temperature. Using the same assumptions for the growth of $\mathrm{EuO}$ on single crystal diamond substrates, the thermal expansion mismatch between $\mathrm{EuO}$ and single-crystal diamond would lead to an in-plane tensile strain of $0.7 \%$ and an out-of-plane lattice spacing of $5.123 \AA$ for the $25^{\circ} \mathrm{C}$ XRD measurement. The measured out-of-plane lattice spacings $(5.137 \AA$ and $5.135 \AA$, respectively) implies that the EuO film was not fully relaxed at the growth temperature, but was clamped during cooling. Alternatively, the film was fully relaxed at the growth temperature, but not fully clamped to the substrate during cooling.

${ }^{44}$ T. Mairoser, F. Loder, A. Melville, D. G. Schlom, and A. Schmehl, Phys. Rev. B 87, 014416 (2013). 